\title{
Monitoring intake of free sugars: benefits of assessing the usual intake distribution of population groups
}

\author{
Niina Kaartinen, Heli Tapanainen, Liisa Valsta and Satu Männistö \\ National Institute for Health and Welfare, Helsinki, Finland
}

\begin{abstract}
Free sugars, especially in the form of sugar-sweetened beverages, easily provide excess energy leading to increased risk of obesity and various NCDs. Free sugar intake below $10 \mathrm{E} \%$ is a public health nutrition target established in international (WHO) and many national nutrition recommendations across Europe. However, representative population-based data on free sugar intakes from the overall diet (i.e. free sugars from both liquid and solid foods) remain sparse. Besides mean intakes, the usual intake distributions of free sugar intake overall and in sociodemographic groups are infrequently reported. We aimed to study the intake of free sugars in the Finnish adults, and evaluate the proportion of the population reaching the recommendation. We used the data of the nationally representative FinDiet 2017 Survey consisting of two non-consecutive 24-hour dietary recalls obtained from 1655 subjects aged 18-74 years (53\% participation rate). Weighing factors were used to correct non-participation bias. Assessment of daily free sugar intake was based on a food disaggregation procedure utilizing the national food composition database Fineli ${ }^{\circledR}$ (including standard recipes and ingredient level values for all different monosaccharides and disaccharides). Usual intake distribution modelling was performed using the SPADE program (RIVM, the Netherlands). We used SAS version 9.3 and R statistical software version 3.4 .1 for the analyses. $\mathrm{P}$-values $<0.05$ were considered statistically significant.The mean intake of free sugars was 9.2 E\% in men (n = 780) (P25: 6.4 E\%, P50: $8.7 \mathrm{E} \%$, P75: $11.5 \mathrm{E} \%)$, and $9.3 \mathrm{E} \%$ in women $(\mathrm{n}=875)(\mathrm{P} 25: 6.6 \mathrm{E} \%, \mathrm{P} 50$ : $8.8 \mathrm{E} \%, \mathrm{P} 75$ : $11.4 \mathrm{E} \%)$. However, the recommendation (free sugars $<10 \mathrm{E} \%$ ) was not reached by $37 \%$ of the population. Across the age groups (18-24, 25-44, 45-64, 65-74 years, respectively), the proportion of not reaching the recommendation varied between $28 \%-40 \%$ (men) and $44 \%-32 \%$ (women). Regarding educational groups (categories: low, middle, high), mean free sugar intake in men was higher in the lowest educational group $(9.9 \mathrm{E} \%)$ compared with the highest educational group $(8.8 \mathrm{E} \%)(P$-value: 0.01$)$. In women, free sugar intake did not differ between the educational groups. Overall, despite mean free sugar intakes below $10 \mathrm{E} \%$, at least one third of Finnish adults did not reach the recommendation. Population groups with high free sugar intakes and the determinants of intake deserve further studying. Such information is needed in targeting interventions and policy actions to improve and sustain an optimal free sugar intake in all population groups.
\end{abstract}

\section{Conflict of Interest}

There is no conflict of interest 